\title{
Round Security and Super-Pseudorandomness of MISTY Type Structure
}

\author{
Tetsu Iwata, Tomonobu Yoshino, Tomohiro Yuasa, and Kaoru Kurosawa \\ Department of Communication and Integrated Systems, \\ Tokyo Institute of Technology \\ 2-12-1 O-okayama, Meguro-ku, Tokyo 152-8552, Japan \\ \{tez, kurosawa\}@ss.titech.ac.jp
}

\begin{abstract}
The security of an iterated block cipher heavily depends on its structure as well as each round function. Matsui showed that MISTY type structure is faster and more robust than Feistel structure on linear cryptanalysis and differential cryptanalysis. On the other hand, Luby and Rackoff proved that the four round Feistel structure is superpseudorandom if each round function $f_{i}$ is a random function. This paper proves that the five round MISTY type structure is super-pseudorandom. We also characterize its round security.
\end{abstract}

\section{Introduction}

The security of an iterated block cipher heavily depends on its structure as well as each round function. There are some well known structures of iterated block ciphers, Feistel structure (for example, DES), MISTY type structure, IDEA type structure and etc. For Feistel structure, Nyberg and Knudsen [7] showed that if each round function is secure against linear cryptanalysis and differential cryptanalysis, then the whole block cipher is immune to both attacks. Matsui showed that MISTY type structure is faster and more robust than Feistel structure on linear cryptanalysis and differential cryptanalysis [45].

Pseudorandomness is also an important cryptographic criterion of iterated block ciphers. This approach studies the pseudorandomness of the block cipher by assuming that each round function is ideally random. We say that a block cipher is pseudorandom if it is secure against chosen plaintext attack, where the adversary has access only to the forward direction of the block cipher. It is said to be super-pseudorandom if it is secure under both chosen plaintext and chosen ciphertext attacks, where the adversary has access to both directions of the block cipher.

The super-pseudorandomness of Feistel structure has been studied extensively so far. Luby and Rackoff proved that the three round Feistel structure is pseudorandom and the four round Feistel structure is super-pseudorandom if each round function $f_{i}$ is a random function [2]. Patarin gave an alternate proof [89]. Lucks showed that the three round Feistel structure is pseudorandom even if the first round function $f_{1}$ is an XOR-universal hash function (not 
necessarily random) [3]. Naor and Reingold showed that the four round Feistel structure is super-pseudorandom even if the first and the last round functions $f_{1}$ and $f_{4}$ are XOR-universal [6]. Finally, Ramzan and Reyzin showed that the four round Feistel structure is super-pseudorandom even if the adversary has oracle access to the second and the third round functions $f_{2}$ and $f_{3}$, but not super-pseudorandom if the adversary has oracle access to the first or the last round function, $f_{1}$ or $f_{4}[10$.

However, only a little is known about the super-pseudorandomness of MISTY type structure. Sakurai and Zheng showed that the three round MISTY type structure is not pseudorandom, and the four round MISTY type structure is not super-pseudorandom [1]. On the other hand, it is not known if the five round MISTY type structure is super-pseudorandom [11.

This paper characterizes the super-pseudorandomness of the five round MISTY type structure. We prove that the five round MISTY type structure is super-pseudorandom even if:

1. The first, second and the last round functions, $p_{1}, p_{2}$ and $p_{5}$, are XORuniversal permutations. This holds even if the adversary has oracle access to the third and fourth round functions $p_{3}$ and $p_{4}$.

2. The first and the last round functions, $p_{1}$ and $p_{5}$, are XOR-universal. This holds even if the adversary has oracle access to the second, third and fourth round functions, $p_{2}, p_{3}$ and $p_{4}$.

We also show that it is not super-pseudorandom if the adversary is allowed to have oracle access to the first or the last round function, $p_{1}$ or $p_{5}$.

Intuitively, our results can be stated as follows. The five round MISTY type structure is super-pseudorandom if: (1) the first and the last rounds have secrecy and only weak randomness, (2) the third and fourth rounds have strong randomness and no secrecy, and (3) the second round has secrecy and only weak randomness, or no secrecy and strong randomness.

To derive our positive results, we use Patarin's approach [8,9] while Ramzan and Reyzin [10] used the approach of Naor and Reingold [6].

Related works: About pseudorandomness (but not super-pseudorandomness) Sugita showed that the four round MISTY type structure is pseudorandom [12], and the five round recursive MISTY type structure is pseudorandom [13].

\section{Preliminaries}

\subsection{Notation}

For a bit string $x \in\{0,1\}^{2 n}$, we denote the first (left) $n$ bits of $x$ by $x_{L}$ and the last (right) $n$ bits of $x$ by $x_{R}$. If $S$ is a probability space, then $s \stackrel{R}{\leftarrow} S$ denotes the process of picking an element from $S$ according to the underlying probability distribution. (Unless otherwise specified,) The underlying distribution is assumed to be uniform. 
Denote by $F_{n}$ the set of all functions from $\{0,1\}^{n}$ to $\{0,1\}^{n}$, which consists of $2^{n \cdot 2^{n}}$ in total. Similarly, denote by $P_{n}$ the set of all permutations from $\{0,1\}^{n}$ to $\{0,1\}^{n}$, which consists of $\left(2^{n}\right)$ ! in total. By a finite function (or permutation) family $\mathcal{F}$, we denote a set of functions with common domain and common range. We call a finite function (or permutation) family keyed if every function in it can be specified by a key $s k$. We denote the function given by $s k$ as $f_{s k}$. We assume that given $s k$, it is possible to efficiently evaluate $f_{s k}$ at any point (as well as $f_{s k}^{-1}$ in case of a keyed permutation family). For a given keyed function family, a key can be any string from $\{0,1\}^{s}$, where $s$ is known as "key length." For functions $f$ and $g, g \circ f$ denotes the function $x \mapsto g(f(x))$.

\subsection{Super-Pseudorandomness}

We are now ready to define a secure block cipher, or what Luby and Rackoff called a super-pseudorandom permutation [2]. The super-pseudorandomness of a keyed permutation family $\mathcal{F}$ over $\{0,1\}^{n}$ captures its computational indistinguishability from $P_{n}$, where the adversary is given access to both directions of the permutation. In other words, it measures security of a block cipher against chosen plaintext and chosen ciphertext attacks.

Our adaptive adversary $\mathcal{M}$ is modeled as a Turing machine that has blackbox access to some number $k$ of oracles, each of which computes some specified function. If $\left(f_{1}, \ldots, f_{k}\right)$ is a $k$-tuple of functions, then $\mathcal{M}^{f_{1}, \ldots, f_{k}}$ denotes a $k$-oracle adversary who is given black-box access to each of the functions $f_{1}, \ldots, f_{k}$. The computational power of $\mathcal{M}$ is unlimited, but the total number of oracle calls is limited to a parameter $m$.

Definition 2.1. (Advantage, sprp). Let a block cipher $\mathcal{F}$ be a keyed permutation family over $\{0,1\}^{n}$ with key length s. Let $\mathcal{M}$ be a 2-oracle adversary. Then we define $\mathcal{M}$ 's advantage as

$$
\operatorname{Adv}_{\mathcal{F}}^{\text {sprp }}(\mathcal{M}) \stackrel{\text { def }}{=}\left|p_{f}-p_{R}\right|
$$

where

$$
\left\{\begin{array}{l}
p_{f} \stackrel{\text { def }}{=} \operatorname{Pr}\left(\mathcal{M}^{f_{s k}, f_{s k}^{-1}}\left(1^{n}\right)=1 \mid s k \stackrel{R}{\leftarrow}\{0,1\}^{s}\right) \\
p_{R} \stackrel{\text { def }}{=} \operatorname{Pr}\left(\mathcal{M}^{R, R^{-1}}\left(1^{n}\right)=1 \mid R \stackrel{R}{\leftarrow} P_{n}\right)
\end{array}\right.
$$

Definition 2.2. (Super-pseudorandom permutation family). A block cipher $\mathcal{F}$ is super-pseudorandom if $\operatorname{Adv}_{\mathcal{F}}^{\text {sprp }}(\mathcal{M})$ is negligible for any 2-oracle adversary $\mathcal{M}$.

\subsection{MISTY Type Permutation 4,5}

Matsui proposed MISTY [45], which is faster and more robust than Feistel structure on linear cryptanalysis and differential cryptanalysis. 
Definition 2.3. (The basic MISTY type permutation). Let $x \in\{0,1\}^{2 n}$. For any permutation $p \in P_{n}$, define the basic MISTY type permutation, $M_{p} \in$ $P_{2 n}$ as $M_{p}(x) \stackrel{\text { def }}{=}\left(x_{R}, p\left(x_{L}\right) \oplus x_{R}\right)$. Note that it is a permutation since $M_{p}^{-1}(x)=$ $\left(p^{-1}\left(x_{L} \oplus x_{R}\right), x_{L}\right)$.

Definition 2.4. (The $r$ round MISTY type permutation, $\psi$ ). Let $r \geq 1$ be an integer, $p_{1}, \ldots, p_{r} \in P_{n}$ be permutations. Define the $r$ round MISTY type permutation $\psi\left(p_{1}, \ldots, p_{r}\right) \in P_{2 n}$ as $\psi\left(p_{1}, \ldots, p_{r}\right) \stackrel{\text { def }}{=} \rho \circ M_{p_{r}} \circ \cdots \circ M_{p_{1}}$, where $\rho\left(x_{L}, x_{R}\right)=\left(x_{R}, x_{L}\right)$ for $x \in\{0,1\}^{2 n}$.

See Fig. 1 (the four round Feistel permutation) and Fig. 2 (the five round MISTY type permutation) for illustrations. Note that $p_{i}$ in Fig. 2 is a permutation whereas $f_{i}$ in Fig. 1 is just a function. For simplicity, the left and right swaps are omitted.

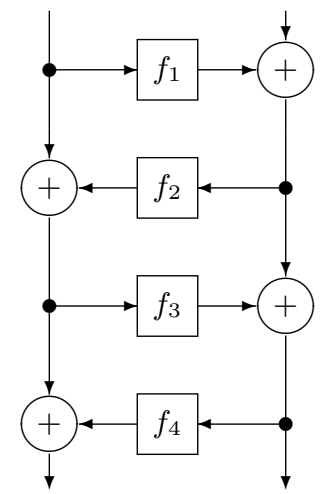

Fig. 1. Feistel permutation

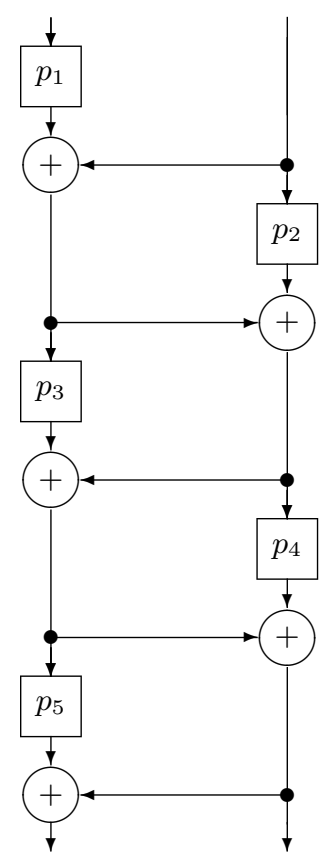

Fig. 2. MISTY type permutation

\subsection{Round Security of the Five Round MISTY Type Permutation}

The round security model of a block cipher was introduced by Ramzan and Reyzin [10]. In the round security model, the adversary is allowed to have oracle access to some subset $K$ of round functions, and the advantage additionally depends on $K$. 
Definition 2.5. (Round security of the five round MISTY type permutation). Let $p_{i}$ be a permutation drawn from a keyed permutation family $\mathcal{P}_{i}$ over $\{0,1\}^{n}$ with key length $s_{i}$, for $i=1, \ldots, 5$. Let $\psi\left(p_{1}, \ldots, p_{5}\right)$ be the five round MISTY type permutation, and $\Psi$ be the set of $\psi\left(p_{1}, \ldots, p_{5}\right)$ with key length $s=s_{1}+\cdots+s_{5}$ (the key sk for $\psi\left(p_{1}, \ldots, p_{5}\right)$ is simply the concatenation of keys for $\left.p_{1}, \ldots, p_{5}\right)$. Fix some subset $\mathcal{K}=\left\{\Pi^{1}, \ldots, \Pi^{k}\right\}$ of the set $\left\{\mathcal{P}_{1}, \mathcal{P}_{1}^{-1}, \ldots, \mathcal{P}_{5}, \mathcal{P}_{5}^{-1}\right\}$, and let $\mathcal{M}$ be a $(k+2)$-oracle adversary. Let $K \stackrel{\text { def }}{=}\left\{\pi_{s k}^{1}, \ldots, \pi_{s k}^{k}\right\}$, where $\pi_{s k}^{i} \in \Pi^{i}$ for $1 \leq i \leq k$. Then we define $\mathcal{M}$ 's advantage as

$$
\operatorname{Adv}_{\Psi ; \mathcal{K}}^{\text {sprp }}(\mathcal{M}) \stackrel{\text { def }}{=}\left|p_{\psi}-p_{R}\right|
$$

where

$$
\left\{\begin{array}{l}
p_{\psi} \stackrel{\text { def }}{=} \operatorname{Pr}\left(\mathcal{M}^{\psi_{s k}, \psi_{s k}^{-1}, \pi_{s k}^{1}, \ldots, \pi_{s k}^{k}}\left(1^{2 n}\right)=1 \mid s k \stackrel{R}{\leftarrow}\{0,1\}^{s}\right) \\
p_{R} \stackrel{\text { def }}{=} \operatorname{Pr}\left(\mathcal{M}^{R, R^{-1}, \pi_{s k}^{1}, \ldots, \pi_{s k}^{k}}\left(1^{2 n}\right)=1 \mid R \stackrel{R}{\leftarrow} P_{2 n}, s k \stackrel{R}{\leftarrow}\{0,1\}^{s}\right)
\end{array}\right.
$$

\subsection{Uniform $\epsilon$-XOR Universal Permutation}

Our definition follows from those given in [1|10:14.

Definition 2.6. Let $H_{n}$ be a keyed permutation family over $\{0,1\}^{n}$. Denote by $\# H_{n}$ the size of $H_{n} . H_{n}$ is uniform $\epsilon$-XOR universal provided that the following two conditions are satisfied:

1. for any element $x \in\{0,1\}^{n}$ and any element $y \in\{0,1\}^{n}$, there exist exactly $\frac{\# H_{n}}{2^{n}}$ permutations $h \in H_{n}$ such that $h(x)=y$.

2. for any two distinct elements $x, x^{\prime} \in\{0,1\}^{n}$ and any element $y \in\{0,1\}^{n}$, there exist at most $\epsilon \# H_{n}$ permutations $h \in H_{n}$ such that $h(x) \oplus h\left(x^{\prime}\right)=y$.

Let $f_{a, b}(x) \stackrel{\text { def }}{=} a \cdot x+b$ over $\operatorname{GF}\left(2^{n}\right)$, where $a \neq 0$. Then $\left\{f_{a, b}(x)\right\}$ is uniform $\frac{1}{2^{n}-1}$-XOR universal.

We will use the phrase " $h$ is an uniform $\epsilon$-XOR universal permutation" to mean that " $h$ is drawn uniformly from an uniform $\epsilon$-XOR universal permutation family."

\section{Round Security of MISTY Type Permutation}

\subsection{Negative Result}

In this section, we show that $\psi\left(p_{1}, p_{2}, p_{3}, p_{4}, p_{5}\right)$ is not super-pseudorandom if the adversary is allowed to have oracle access to $\left\{p_{1}, p_{1}^{-1}\right\}$ or $\left\{p_{5}^{-1}\right\}$. This means that, we require secrecy in the first and the last rounds if the cipher is secure.

Theorem 3.1. Let $p_{1}, p_{2}, p_{3}, p_{4}, p_{5} \in P_{n}$ be random permutations. Let $\psi=$ $\psi\left(p_{1}, p_{2}, p_{3}, p_{4}, p_{5}\right)$, and $R \in P_{2 n}$ be a random permutation. Suppose that $K$ contains at least one of $\left\{p_{1}, p_{1}^{-1}\right\}$ or $\left\{p_{5}^{-1}\right\}$. Then there exists an oracle adversary $\mathcal{M}$ such that

$$
\operatorname{Adv}_{\Psi ; \mathcal{K}}^{\text {sprp }}(\mathcal{M}) \geq 1-\frac{2}{2^{n}}
$$


Proof. Let $\mathcal{O}=R$ or $\psi$. First, suppose that $\mathcal{M}$ has oracle access to $\mathcal{O}, \mathcal{O}^{-1}, p_{1}$ and $p_{1}^{-1}$. Consider the following $\mathcal{M}$ :

1. Pick $X, A, A^{\prime} \in\{0,1\}^{n}$ such that $A \neq A^{\prime}$ arbitrarily.

2. Ask $(A, A \oplus X)$ to $\mathcal{O}^{-1}$ and obtain $(C, D)$.

3. Ask $\left(A^{\prime}, A^{\prime} \oplus X\right)$ to $\mathcal{O}^{-1}$ and obtain $\left(C^{\prime}, D^{\prime}\right)$.

4. Ask $C$ to $p_{1}$ and obtain $E$.

5. Ask $C^{\prime}$ to $p_{1}$ and obtain $E^{\prime}$.

6. Ask $D \oplus D^{\prime} \oplus E$ to $p_{1}^{-1}$ and obtain $F$.

7. Ask $D \oplus D^{\prime} \oplus E^{\prime}$ to $p_{1}^{-1}$ and obtain $F^{\prime}$.

8. Ask $\left(F^{\prime}, D\right)$ to $\mathcal{O}$ and obtain $(S, T)$.

9. Ask $\left(F, D^{\prime}\right)$ to $\mathcal{O}$ and obtain $\left(S^{\prime}, T^{\prime}\right)$.

10. Output " 1 " if and only if $S \oplus T=S^{\prime} \oplus T^{\prime}$.

If $\mathcal{O}=\psi$, then $X$ is the output of $p_{5}$ at step 2 and step 3. Hence the input to $p_{5}$ at step 2 is equal to that of step 3 . Therefore, from step 4 and step 5 , we have

$$
p_{2}(D) \oplus D \oplus E \oplus p_{3}(D \oplus E)=p_{2}\left(D^{\prime}\right) \oplus D^{\prime} \oplus E^{\prime} \oplus p_{3}\left(D^{\prime} \oplus E^{\prime}\right)
$$

In step 8, the output of $p_{1}$ is equal to $D \oplus D^{\prime} \oplus E^{\prime}$ from step 7 . Therefore, the input to $p_{5}$ is equal to $p_{2}(D) \oplus D^{\prime} \oplus E^{\prime} \oplus p_{3}\left(D^{\prime} \oplus E^{\prime}\right)$ in step 8. Similarly, in step 9 , the input to $p_{5}$ is equal to $p_{2}\left(D^{\prime}\right) \oplus D \oplus E \oplus p_{3}(D \oplus E)$. Then from eq.(1), we see that the inputs to $p_{5}$ are equal in step 8 and step 9 . Hence we have $p_{\psi}=1$.

If $\mathcal{O}=R$, we have $p_{R} \leq \frac{2}{2^{n}}$.

Next suppose that $\mathcal{M}$ has oracle access to $\mathcal{O}, \mathcal{O}^{-1}$ and $p_{5}^{-1}$. Consider the following $\mathcal{M}$ :

1. Pick $X, B, B^{\prime} \in\{0,1\}^{n}$ such that $B \neq B^{\prime}$ arbitrarily.

2. Ask $B$ to $p_{5}^{-1}$ and obtain $A$.

3. Ask $B^{\prime}$ to $p_{5}^{-1}$ and obtain $A^{\prime}$.

4. Ask $(X, X \oplus B)$ to $\mathcal{O}^{-1}$ and obtain $(C, D)$.

5. Ask $\left(A \oplus A^{\prime} \oplus B \oplus B^{\prime} \oplus X, A \oplus A^{\prime} \oplus B \oplus X\right)$ to $\mathcal{O}^{-1}$ and obtain $\left(C^{\prime}, D^{\prime}\right)$.

6. Ask $\left(C, D^{\prime}\right)$ to $\mathcal{O}$ and obtain $(E, F)$.

7. Ask $\left(C^{\prime}, D\right)$ to $\mathcal{O}$ and obtain $\left(E^{\prime}, F^{\prime}\right)$.

8. Ask $E \oplus F$ to $p_{5}^{-1}$ and obtain $H$.

9. Ask $E^{\prime} \oplus F^{\prime}$ to $p_{5}^{-1}$ and obtain $H^{\prime}$.

10. Output " 1 " if and only if $H \oplus F=H^{\prime} \oplus F^{\prime}$.

If $\mathcal{O}=\psi$, then $A \oplus B \oplus X$ is the outputs of $p_{4}$ at step 4 and step 5 . Then the input to $p_{4}$ at step 6 is equal to that of step 7 . Hence we have $p_{\psi}=1$.

If $\mathcal{O}=R$, we have $p_{R} \leq \frac{2}{2^{n}}$.

\subsection{Positive Result 1}

Let $h_{1}, h_{2}, h_{3} \in H_{n}$ be uniform $\epsilon$-XOR universal permutations and $p \in P_{n}$ be a random permutation. Let $\psi=\psi\left(h_{1}, h_{2}, p, p, h_{3}^{-1}\right)$, and $R \in P_{2 n}$ be a random permutation. Define $K=\left\{p, p^{-1}\right\}$. 
Lemma 3.1. Let $m_{0}$ and $m_{1}$ be integers. Choose $x^{(i)} \in\{0,1\}^{2 n}$ and $y^{(i)} \in$ $\{0,1\}^{2 n}$ for $1 \leq i \leq m_{0}$ arbitrarily in such a way that $x^{(i)}$ are all distinct and $y^{(i)}$ are all distinct. Choose $X^{(i)} \in\{0,1\}^{n}$ and $Y^{(i)} \in\{0,1\}^{n}$ for $1 \leq i \leq m_{1}$ arbitrarily in such a way that $X^{(i)}$ are all distinct and $Y^{(i)}$ are all distinct.

Then the number of $\left(h_{1}, h_{2}, p, h_{3}\right)$ such that

$$
\left.\begin{array}{l}
\psi\left(x^{(i)}\right)=y^{(i)} \text { for } 1 \leq \forall i \leq m_{0}, \text { and } \\
p\left(X^{(i)}\right)=Y^{(i)} \text { for } 1 \leq \forall i \leq m_{1}
\end{array}\right\}
$$

is at least

$$
\left(\# H_{n}\right)^{3}\left(2^{n}-2 m_{0}-m_{1}\right) !\left(1-\epsilon \cdot m_{0}\left(3 m_{0}-2\right)-\frac{2 m_{0}\left(m_{0}+2 m_{1}\right)}{2^{n}}\right) .
$$

A proof is given in the next section.

Theorem 3.2. For any 4-oracle adversary $\mathcal{M}$ that makes at most $m$ queries in total,

$$
\operatorname{Adv}_{\Psi ; \mathcal{K}}^{\text {sprp }}(\mathcal{M}) \leq \epsilon \cdot m(3 m-2)+\frac{4 m^{2}}{2^{n}}
$$

Proof. Let $\mathcal{O}=R$ or $\psi$. The 4-oracle adversary $\mathcal{M}$ has oracle access to $\mathcal{O}, \mathcal{O}^{-1}$, $p$ and $p^{-1}$. Assume that $\mathcal{M}$ makes $m_{0}$ queries to $\mathcal{O}$ or $\mathcal{O}^{-1}$, and $m_{1}$ queries to $p$ or $p^{-1}$, where $m=m_{0}+m_{1}$.

Let $q^{(1)}, \ldots, q^{\left(m_{0}\right)} \in\{0,1\}^{2 n}$ be bit strings that $\mathcal{M}$ asks to $\mathcal{O}$ or $\mathcal{O}^{-1}$, and let $a^{(1)}, \ldots, a^{\left(m_{0}\right)} \in\{0,1\}^{2 n}$ be the answers that $\mathcal{M}$ obtains. Let $Q^{(1)}, \ldots, Q^{\left(m_{1}\right)} \in$ $\{0,1\}^{n}$ be bit strings that $\mathcal{M}$ asks to $p$ or $p^{-1}$, and let $A^{(1)}, \ldots, A^{\left(m_{1}\right)} \in\{0,1\}^{n}$ be the answers that $\mathcal{M}$ obtains.

Let

$$
\begin{gathered}
\left(x^{(i)}, y^{(i)}\right)= \begin{cases}\left(q^{(i)}, a^{(i)}\right) & \text { if } \mathcal{O}\left(q^{(i)}\right)=a^{(i)} \\
\left(a^{(i)}, q^{(i)}\right) & \text { if } \mathcal{O}^{-1}\left(q^{(i)}\right)=a^{(i)},\end{cases} \\
\left(X^{(i)}, Y^{(i)}\right)= \begin{cases}\left(Q^{(i)}, A^{(i)}\right) & \text { if } p\left(Q^{(i)}\right)=A^{(i)} \\
\left(A^{(i)}, Q^{(i)}\right) & \text { if } p^{-1}\left(Q^{(i)}\right)=A^{(i)} .\end{cases}
\end{gathered}
$$

That is,

$$
\mathcal{O}\left(x^{(i)}\right)=y^{(i)} \text { for } 1 \leq i \leq m_{0} \text {, and } p\left(X^{(i)}\right)=Y^{(i)} \text { for } 1 \leq i \leq m_{1} .
$$

Without loss of generality, we assume that $x^{(i)}$ are all distinct, $y^{(i)}$ are all distinct, $X^{(i)}$ are all distinct and $Y^{(i)}$ are all distinct.

Suppose that $\mathcal{M}$ has obtained $a^{(1)}, \ldots, a^{(i)}$ and $A^{(1)}, \ldots, A^{(j)}$ from the oracles at some point. Then the next behavior of $\mathcal{M}$ is completely determined by $a^{(1)}, \ldots, a^{(i)}$ and $A^{(1)}, \ldots, A^{(j)}$. Therefore, the final output of $\mathcal{M}(0$ or 1$)$ depends only on $a \stackrel{\text { def }}{=}\left(a^{(1)}, \ldots, a^{\left(m_{0}\right)}\right)$ and $A \stackrel{\text { def }}{=}\left(A^{(1)}, \ldots, A^{\left(m_{1}\right)}\right)$. Hence denote by $\mathcal{C}_{\mathcal{M}}(a, A)$ the final output of $\mathcal{M}$.

Let $\mathcal{B} \stackrel{\text { def }}{=}\left\{(a, A) \mid \mathcal{C}_{\mathcal{M}}(a, A)=1\right\}$ and $N \stackrel{\text { def }}{=} \# \mathcal{B}$. 
Evaluation of $p_{R}$. From the definition of $p_{R}$, we have

$$
p_{R}=\operatorname{Pr}_{R, p}\left(\mathcal{M}^{R, R^{-1}, p, p^{-1}}\left(1^{2 n}\right)=1\right)=\frac{\#\left\{(R, p) \mid \mathcal{M}^{R, R^{-1}, p, p^{-1}}\left(1^{2 n}\right)=1\right\}}{\left(2^{2 n}\right) !\left(2^{n}\right) !}
$$

We say that $(R, p)$ is compatible with $(a, A)$ if the $\left(R, R^{-1}\right)$ oracles answer $a$ and the $\left(p, p^{-1}\right)$ oracles answer $A$. More precisely, $(R, p)$ is compatible with $(a, A)$ if

$$
R\left(x^{(i)}\right)=y^{(i)} \text { for } 1 \leq i \leq m_{0} \text { and } p\left(X^{(i)}\right)=Y^{(i)} \text { for } 1 \leq i \leq m_{1},
$$

where $x^{(i)}, y^{(i)}, X^{(i)}, Y^{(i)}$ are defined by eq.(3) and eq.(4) from (a,A). For each $(a, A) \in \mathcal{B}$, the number of $(R, p)$ which is compatible with $(a, A)$ is exactly $\left(2^{2 n}-m_{0}\right) !\left(2^{n}-m_{1}\right) !$. Therefore, we have

$$
\begin{aligned}
p_{R} & =\sum_{(a, A) \in \mathcal{B}} \frac{\#\{(R, p) \mid(R, p) \text { is compatible with }(a, A)\}}{\left(2^{2 n}\right) !\left(2^{n}\right) !} \\
& =\sum_{(a, A) \in \mathcal{B}} \frac{\#\{(R, p) \mid(R, p) \text { satisfying ([5) }\}}{\left(2^{2 n}\right) !\left(2^{n}\right) !} \\
& =N \cdot \frac{\left(2^{2 n}-m_{0}\right) !\left(2^{n}-m_{1}\right) !}{\left(2^{2 n}\right) !\left(2^{n}\right) !} .
\end{aligned}
$$

Evaluation of $p_{\psi}$. From the definition of $p_{\psi}$, we have

$$
\begin{aligned}
p_{\psi} & =\operatorname{Pr}_{h_{1}, h_{2}, p, h_{3}}\left(\mathcal{M}^{\psi, \psi^{-1}, p, p^{-1}}\left(1^{2 n}\right)=1\right) \\
& =\frac{\#\left\{\left(h_{1}, h_{2}, p, h_{3}\right) \mid \mathcal{M}^{\psi, \psi^{-1}, p, p^{-1}}\left(1^{2 n}\right)=1\right\}}{\left(\# H_{n}\right)^{3}\left(2^{n}\right) !} .
\end{aligned}
$$

Similarly to $p_{R}$, we have

$$
p_{\psi}=\sum_{(a, A) \in \mathcal{B}} \frac{\#\left\{\left(h_{1}, h_{2}, p, h_{3}\right) \mid\left(h_{1}, h_{2}, p, h_{3}\right) \text { satisfying (2) }\right\}}{\left(\# H_{n}\right)^{3}\left(2^{n}\right) !} .
$$

Then from Lemma 3.1 we obtain that

$$
\begin{aligned}
p_{\psi} & \geq \sum_{(a, A) \in \mathcal{B}} \frac{\left(\# H_{n}\right)^{3}\left(2^{n}-2 m_{0}-m_{1}\right) !\left(1-\epsilon \cdot m_{0}\left(3 m_{0}-2\right)-\frac{2 m_{0}\left(m_{0}+2 m_{1}\right)}{2^{n}}\right)}{\left(\# H_{n}\right)^{3}\left(2^{n}\right) !} \\
& =N \frac{\left(2^{n}-2 m_{0}-m_{1}\right) !}{\left(2^{n}\right) !}\left(1-\epsilon \cdot m_{0}\left(3 m_{0}-2\right)-\frac{2 m_{0}\left(m_{0}+2 m_{1}\right)}{2^{n}}\right) \\
& =p_{R} \frac{\left(2^{2 n}\right) !\left(2^{n}-2 m_{0}-m_{1}\right) !}{\left(2^{2 n}-m_{0}\right) !\left(2^{n}-m_{1}\right) !}\left(1-\epsilon \cdot m_{0}\left(3 m_{0}-2\right)-\frac{2 m_{0}\left(m_{0}+2 m_{1}\right)}{2^{n}}\right) .
\end{aligned}
$$


Since $\frac{\left(2^{2 n}\right) !\left(2^{n}-2 m_{0}-m_{1}\right) !}{\left(2^{2 n}-m_{0}\right) !\left(2^{n}-m_{1}\right) !} \geq 1$ (This can be shown easily by an induction on $\left.m_{0}\right)$, we have

$$
\begin{aligned}
p_{\psi} & \geq p_{R}\left(1-\epsilon \cdot m_{0}\left(3 m_{0}-2\right)-\frac{2 m_{0}\left(m_{0}+2 m_{1}\right)}{2^{n}}\right) \\
& \geq p_{R}-\epsilon \cdot m_{0}\left(3 m_{0}-2\right)-\frac{2 m_{0}\left(m_{0}+2 m_{1}\right)}{2^{n}} \\
& \geq p_{R}-\epsilon \cdot m(3 m-2)-\frac{4 m^{2}}{2^{n}} .
\end{aligned}
$$

Applying the same argument to $1-p_{\psi}$ and $1-p_{R}$ yields that

$$
1-p_{\psi} \geq 1-p_{R}-\epsilon \cdot m(3 m-2)-\frac{4 m^{2}}{2^{n}}
$$

Finally, (6) and (17) give $\left|p_{\psi}-p_{R}\right| \leq \epsilon \cdot m(3 m-2)+\frac{4 m^{2}}{2^{n}}$.

\subsection{Positive Result 2}

Let $h_{1}, h_{2} \in H_{n}$ be uniform $\epsilon$-XOR-universal permutations, $p_{1}, p_{2}, p_{3} \in P_{n}$ be random permutations, $\psi=\psi\left(h_{1}, p_{1}, p_{2}, p_{3}, h_{2}^{-1}\right)$, and $R \in P_{2 n}$ be a random permutation. Define $K=\left\{p_{1}, p_{1}^{-1}, p_{2}, p_{2}^{-1}, p_{3}, p_{3}^{-1}\right\}$.

Lemma 3.2. Let $m_{0}, m_{1}, m_{2}, m_{3}$ be integers such that $m=m_{0}+m_{1}+m_{2}+m_{3}$. Choose $x^{(i)} \in\{0,1\}^{2 n}$ and $y^{(i)} \in\{0,1\}^{2 n}$ for $1 \leq i \leq m_{0}$ arbitrarily in such $a$ way that $x^{(i)}$ are all distinct and $y^{(i)}$ are all distinct. Similarly, for $1 \leq l \leq 3$, choose $X_{l}^{(i)} \in\{0,1\}^{n}$ and $Y_{l}^{(i)} \in\{0,1\}^{n}$ for $1 \leq i \leq m_{l}$ arbitrarily in such $a$ way that $X_{l}^{(i)}$ are all distinct, $Y_{l}^{(i)}$ are all distinct and

$$
X_{1}^{(i)} \oplus Y_{1}^{(i)} \neq X_{1}^{(j)} \oplus Y_{1}^{(j)} \text { for } l=1 \text { and } 1 \leq \forall i<\forall j \leq m_{1}
$$

Then the number of $\left(h_{1}, p_{1}, p_{2}, p_{3}, h_{2}\right)$ such that

$$
\left.\begin{array}{l}
\psi\left(x^{(i)}\right)=y^{(i)} \text { for } 1 \leq \forall i \leq m_{0}, \\
p_{1}\left(X_{1}^{(i)}\right)=Y_{1}^{(i)} \text { for } 1 \leq \forall i \leq m_{1}, \\
p_{2}\left(X_{2}^{(i)}\right)=Y_{2}^{(i)} \text { for } 1 \leq \forall i \leq m_{2}, \text { and } \\
p_{3}\left(X_{3}^{(i)}\right)=Y_{3}^{(i)} \text { for } 1 \leq \forall i \leq m_{3}
\end{array}\right\}
$$

is at least

$$
\begin{aligned}
& \left(2^{n}-m_{1}\right) !\left(2^{n}-m_{2}-m_{0}\right) !\left(2^{n}-m_{3}-m_{0}\right) ! \\
& \quad\left(\# H_{n}\right)^{2}\left(1-2 \epsilon \cdot m_{0}\left(m_{0}-1\right)-\frac{m_{0}(2 m-1)}{2^{n}}\right)
\end{aligned}
$$

A proof is similar to that of Lemma 3.1 
Theorem 3.3. For any 8-oracle adversary $\mathcal{M}$ that makes at most $m$ queries in total,

$$
\operatorname{Adv}_{\Psi ; \mathcal{K}}^{\mathrm{sprp}}(\mathcal{M}) \leq 2 \epsilon \cdot m(m-1)+\frac{m(3 m-2)}{2^{n}}
$$

Proof. Let $\mathcal{O}=R$ or $\psi$. The 8 -oracle adversary $\mathcal{M}$ has oracle access to $\mathcal{O}, \mathcal{O}^{-1}$, $p_{1}, p_{1}^{-1}, p_{2}, p_{2}^{-1}, p_{3}$ and $p_{3}^{-1}$. Assume that $\mathcal{M}$ makes $m_{0}$ queries to $\mathcal{O}$ or $\mathcal{O}^{-1}$ and $m_{l}$ queries to $p_{l}$ or $p_{l}^{-1}$ for $1 \leq l \leq 3$, where $m=m_{0}+m_{1}+m_{2}+m_{3}$.

Let $q^{(1)}, \ldots, q^{\left(m_{0}\right)} \in\{0,1\}^{2 n}$ be bit strings that $\mathcal{M}$ asks to $\mathcal{O}$ or $\mathcal{O}^{-1}$, and let $a^{(1)}, \ldots, a^{\left(m_{0}\right)} \in\{0,1\}^{2 n}$ be the answers that $\mathcal{M}$ obtains. For $1 \leq l \leq 3$, let $Q_{l}^{(1)}, \ldots, Q_{l}^{\left(m_{l}\right)} \in\{0,1\}^{n}$ be bit strings that $\mathcal{M}$ asks to $p_{l}$ or $p_{l}^{-1}$, and let $A_{l}^{(1)}, \ldots, A_{l}^{\left(m_{l}\right)} \in\{0,1\}^{n}$ be the answers that $\mathcal{M}$ obtains.

Let

$$
\begin{gathered}
\left(x^{(i)}, y^{(i)}\right)= \begin{cases}\left(q^{(i)}, a^{(i)}\right) & \text { if } \mathcal{O}\left(q^{(i)}\right)=a^{(i)} \\
\left(a^{(i)}, q^{(i)}\right) & \text { if } \mathcal{O}^{-1}\left(q^{(i)}\right)=a^{(i)}\end{cases} \\
\left(X_{l}^{(i)}, Y_{l}^{(i)}\right)= \begin{cases}\left(Q_{l}^{(i)}, A_{l}^{(i)}\right) & \text { if } p_{l}\left(Q_{l}^{(i)}\right)=A_{l}^{(i)} \\
\left(A_{l}^{(i)}, Q_{l}^{(i)}\right) & \text { if } p_{l}^{-1}\left(Q_{l}^{(i)}\right)=A_{l}^{(i)}\end{cases}
\end{gathered}
$$

for $1 \leq l \leq 3$. That is, $\mathcal{O}\left(x^{(i)}\right)=y^{(i)}$ for $1 \leq i \leq m_{0}$ and $p_{l}\left(X_{l}^{(i)}\right)=Y_{l}^{(i)}$ for $1 \leq l \leq 3$ and $1 \leq i \leq m_{l}$.

Without loss of generality, we assume that $x^{(i)}$ are all distinct, $y^{(i)}$ are all distinct, $X_{l}^{(i)}$ are all distinct and $Y_{l}^{(i)}$ are all distinct, for $1 \leq l \leq 3$.

Define $a \stackrel{\text { def }}{=}\left(a^{(1)}, \ldots, a^{\left(m_{0}\right)}\right)$ and $A_{l} \stackrel{\text { def }}{=}\left(A_{l}^{(1)}, \ldots, A_{l}^{\left(m_{l}\right)}\right)$ for $1 \leq l \leq 3$. Then, similarly to the proof of Theorem 3.2 , we can denote by $\mathcal{C}_{\mathcal{M}}\left(a, A_{1}, A_{2}, A_{3}\right)$ the output of $\mathcal{M}(0$ or 1$)$. Let

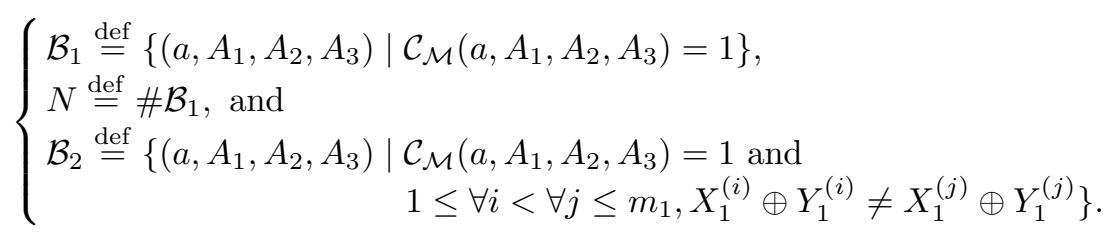

Evaluation of $p_{R}$. From the definition of $p_{R}$, we have

$$
\begin{aligned}
p_{R} & =\operatorname{Pr}_{R, p_{1}, p_{2}, p_{3}}\left(\mathcal{M}^{R, R^{-1}, p_{1}, p_{1}^{-1}, p_{2}, p_{2}^{-1}, p_{3}, p_{3}^{-1}}\left(1^{2 n}\right)=1\right) \\
& =\frac{\#\left\{\left(R, p_{1}, p_{2}, p_{3}\right) \mid \mathcal{M}^{R, R^{-1}, p_{1}, p_{1}^{-1}, p_{2}, p_{2}^{-1}, p_{3}, p_{3}^{-1}}\left(1^{2 n}\right)=1\right\}}{\left(2^{2 n}\right) !\left(\left(2^{n}\right) !\right)^{3}} .
\end{aligned}
$$

Since the number of $\left(R, p_{1}, p_{2}, p_{3}\right)$ such that

$$
\left.\begin{array}{l}
R\left(x^{(i)}\right)=y^{(i)} \text { for } 1 \leq \forall i \leq m_{0}, \\
p_{1}\left(X_{1}^{(i)}\right)=Y_{1}^{(i)} \text { for } 1 \leq \forall i \leq m_{1}, \\
p_{2}\left(X_{2}^{(i)}\right)=Y_{2}^{(i)} \text { for } 1 \leq \forall i \leq m_{2}, \text { and } \\
p_{3}\left(X_{3}^{(i)}\right)=Y_{3}^{(i)} \text { for } 1 \leq \forall i \leq m_{3}
\end{array}\right\}
$$

is exactly $\left(2^{2 n}-m_{0}\right) !\left(2^{n}-m_{1}\right) !\left(2^{n}-m_{2}\right) !\left(2^{n}-m_{3}\right)$ !, we have 


$$
\begin{aligned}
p_{R} & =\sum_{\left(a, A_{1}, A_{2}, A_{3}\right) \in \mathcal{B}_{1}} \frac{\#\left\{\left(R, p_{1}, p_{2}, p_{3}\right) \mid\left(R, p_{1}, p_{2}, p_{3}\right) \text { satisfying (11) }\right\}}{\left(2^{2 n}\right) !\left(\left(2^{n}\right) !\right)^{3}} \\
& =N \cdot \frac{\left(2^{2 n}-m_{0}\right) !\left(2^{n}-m_{1}\right) !\left(2^{n}-m_{2}\right) !\left(2^{n}-m_{3}\right) !}{\left(2^{2 n}\right) !\left(\left(2^{n}\right) !\right)^{3}} .
\end{aligned}
$$

Define $C$ be the total number of possible $\left(a, A_{1}, A_{2}, A_{3}\right)$. Then

$$
C=\frac{\left(2^{2 n}\right) !}{\left(2^{2 n}-m_{0}\right) !} \frac{\left(2^{n}\right) !}{\left(2^{n}-m_{1}\right) !} \frac{\left(2^{n}\right) !}{\left(2^{n}-m_{2}\right) !} \frac{\left(2^{n}\right) !}{\left(2^{n}-m_{3}\right) !} .
$$

Therefore we have $p_{R}=\frac{N}{C}$.

Evaluation of $p_{\psi}$. From the definition of $p_{\psi}$, we have

$$
\begin{aligned}
& p_{\psi}=\operatorname{Pr}_{h_{1}, p_{1}, p_{2}, p_{3}, h_{2}}\left(\mathcal{M}^{\psi, \psi^{-1}, p_{1}, p_{1}^{-1}, p_{2}, p_{2}^{-1}, p_{3}, p_{3}^{-1}}\left(1^{2 n}\right)=1\right) \\
& =\frac{\#\left\{\left(h_{1}, p_{1}, p_{2}, p_{3}, h_{2}\right) \mid \mathcal{M}^{\psi, \psi^{-1}, p_{1}, p_{1}^{-1}, p_{2}, p_{2}^{-1}, p_{3}, p_{3}^{-1}}\left(1^{2 n}\right)=1\right\}}{\left(\# H_{n}\right)^{2}\left(\left(2^{n}\right) !\right)^{3}} .
\end{aligned}
$$

Then

$$
p_{\psi} \geq \sum_{\left(a, A_{1}, A_{2}, A_{3}\right) \in \mathcal{B}_{2}} \frac{\#\left\{\left(h_{1}, p_{1}, p_{2}, p_{3}, h_{2}\right) \mid\left(h_{1}, p_{1}, p_{2}, p_{3}, h_{2}\right) \text { satisfying }(\underline{8})\right\}}{\left(\# H_{n}\right)^{2}\left(\left(2^{n}\right) !\right)^{3}} .
$$

Now we want to evaluate $\# \mathcal{B}_{2}$. Fix any $i$ and $j$ such that $1 \leq i<j \leq m_{1}$. Then the number of $A_{1}=\left(A_{1}^{(1)}, \ldots, A_{1}^{\left(m_{1}\right)}\right)$ which satisfies $X_{1}^{(i)} \oplus Y_{1}^{(i)}=\bar{X}_{1}^{(j)} \oplus$ $Y_{1}^{(j)}$ is exactly $\frac{1}{2^{n}-1} \frac{\left(2^{n}\right) !}{\left(2^{n}-m_{1}\right) !}$, since we have $2^{n}$ choice of $A_{1}^{(i)}$ which uniquely determines $A_{1}^{(j)}$ according to the relation $X_{1}^{(i)} \oplus Y_{1}^{(i)}=X_{1}^{(j)} \oplus Y_{1}^{(j)}$, and other bit strings, $A_{1}^{(l)}$ where $l \neq i, j$, can be arbitrarily, we have $\frac{\left(2^{n}-2\right) !}{\left(2^{n}-m_{1}\right) !}$ choice. Since we have $\left(\begin{array}{c}m_{1} \\ 2\end{array}\right)$ choice of $(i, j)$, the number of $\left(a, A_{1}, A_{2}, A_{3}\right)$ which satisfy

$$
1 \leq \exists i<\exists j \leq m_{1}, X_{1}^{(i)} \oplus Y_{1}^{(i)}=X_{1}^{(j)} \oplus Y_{1}^{(j)}
$$

is at most $\left(\begin{array}{c}m_{1} \\ 2\end{array}\right) \frac{C}{2^{n}-1}$, which is upper bounded by $\frac{m_{1}\left(m_{1}-1\right)}{2^{n}} C$. Then it is clear that $\# \mathcal{B}_{2} \geq N-\frac{m_{1}\left(m_{1}-1\right)}{2^{n}} C$.

Define

$$
D \stackrel{\text { def }}{=}\left(1-2 \epsilon \cdot m_{0}\left(m_{0}-1\right)-\frac{m_{0}(2 m-1)}{2^{n}}\right) \text {. }
$$

Then from Lemma 3.2 we have

$$
\begin{aligned}
p_{\psi} & \geq \sum_{\left(a, A_{1}, A_{2}, A_{3}\right) \in \mathcal{B}_{2}} \frac{\left(2^{n}-m_{1}\right) !\left(2^{n}-m_{2}-m_{0}\right) !\left(2^{n}-m_{3}-m_{0}\right) !}{\left(\left(2^{n}\right) !\right)^{3}} D \\
& \geq\left(N-\frac{m_{1}\left(m_{1}-1\right)}{2^{n}} C\right) \frac{\left(2^{n}-m_{1}\right) !\left(2^{n}-m_{2}-m_{0}\right) !\left(2^{n}-m_{3}-m_{0}\right) !}{\left(\left(2^{n}\right) !\right)^{3}} D \\
& =\left(p_{R}-\frac{m_{1}\left(m_{1}-1\right)}{2^{n}}\right) D \cdot C \frac{\left(2^{n}-m_{1}\right) !\left(2^{n}-m_{2}-m_{0}\right) !\left(2^{n}-m_{3}-m_{0}\right) !}{\left(\left(2^{n}\right) !\right)^{3}} .
\end{aligned}
$$


Since $C \frac{\left(2^{n}-m_{1}\right) !\left(2^{n}-m_{2}-m_{0}\right) !\left(2^{n}-m_{3}-m_{0}\right) !}{\left(\left(2^{n}\right) !\right)^{3}}=\frac{\left(2^{2 n}\right) !\left(2^{n}-m_{2}-m_{0}\right) !\left(2^{n}-m_{3}-m_{0}\right) !}{\left(2^{2 n}-m_{0}\right) !\left(2^{n}-m_{2}\right) !\left(2^{n}-m_{3}\right) !} \geq 1$ (This can be shown easily by an induction on $m_{0}$ ), we have

$$
\begin{aligned}
p_{\psi} & \geq\left(p_{R}-\frac{m_{1}\left(m_{1}-1\right)}{2^{n}}\right)\left(1-2 \epsilon \cdot m_{0}\left(m_{0}-1\right)-\frac{m_{0}(2 m-1)}{2^{n}}\right) \\
& \geq p_{R}-2 \epsilon \cdot m_{0}\left(m_{0}-1\right)-\frac{m_{0}(2 m-1)}{2^{n}}-\frac{m_{1}\left(m_{1}-1\right)}{2^{n}} \\
& \geq p_{R}-2 \epsilon \cdot m(m-1)-\frac{m(3 m-2)}{2^{n}} .
\end{aligned}
$$

Then we have

$$
\left|p_{\psi}-p_{R}\right| \leq 2 \epsilon \cdot m(m-1)+\frac{m(3 m-2)}{2^{n}}
$$

by applying the same argument as was used in Theorem 3.2

\section{Proof of Lemma 3.1}

In $\psi$, we denote by $I_{3} \in\{0,1\}^{n}$, the input to $p$ in the third round, and denote by $O_{3} \in\{0,1\}^{n}$, the output of it. Similarly, $I_{4}, O_{4} \in\{0,1\}^{n}$ are the input and output of $p$ in the fourth round, respectively.

Number of $h_{1}$. First,

- if $x_{L}^{(i)}=x_{L}^{(j)}$, then there exists no $h_{1}$ which satisfies

$$
h_{1}\left(x_{L}^{(i)}\right) \oplus x_{R}^{(i)}=h_{1}\left(x_{L}^{(j)}\right) \oplus x_{R}^{(j)}
$$

since $x_{L}^{(i)}=x_{L}^{(j)}$ implies $x_{R}^{(i)} \neq x_{R}^{(j)}$.

- if $x_{L}^{(i)} \neq x_{L}^{(j)}$, then the number of $h_{1}$ which satisfies (12) is at most $\epsilon \# H_{n}$ from Definition 2.6.

Therefore, the number of $h_{1}$ which satisfies

$$
1 \leq \exists i<\exists j \leq m_{0}, h_{1}\left(x_{L}^{(i)}\right) \oplus x_{R}^{(i)}=h_{1}\left(x_{L}^{(j)}\right) \oplus x_{R}^{(j)}
$$

is at most $\epsilon\left(\begin{array}{c}m_{0} \\ 2\end{array}\right) \# H_{n}$.

Next, the number of $h_{1}$ which satisfies

$$
h_{1}\left(x_{L}^{(i)}\right) \oplus x_{R}^{(i)}=X^{(j)}
$$

is exactly $\frac{\# H_{n}}{2^{n}}$ from Definition 2.6. Therefore, the number of $h_{1}$ which satisfies

$$
1 \leq \exists i \leq m_{0}, 1 \leq \exists j \leq m_{1}, \quad h_{1}\left(x_{L}^{(i)}\right) \oplus x_{R}^{(i)}=X^{(j)}
$$

is at most $\frac{m_{0} m_{1} \# H_{n}}{2^{n}}$.

Then, from (13) and (14), the number of $h_{1}$ which satisfies

$$
\left.\begin{array}{l}
1 \leq \forall i<\forall j \leq m_{0}, \quad h_{1}\left(x_{L}^{(i)}\right) \oplus x_{R}^{(i)} \neq h_{1}\left(x_{L}^{(j)}\right) \oplus x_{R}^{(j)}, \text { and } \\
1 \leq \forall i \leq m_{0}, 1 \leq \forall j \leq m_{1}, \quad h_{1}\left(x_{L}^{(i)}\right) \oplus x_{R}^{(i)} \neq X^{(j)}
\end{array}\right\}
$$


is at least $\# H_{n}-\epsilon\left(\begin{array}{c}m_{0} \\ 2\end{array}\right) \# H_{n}-\frac{m_{0} m_{1} \# H_{n}}{2^{n}}$. Fix $h_{1}$ which satisfies (15) arbitrarily. This implies that $I_{3}^{(1)}, \ldots, I_{3}^{\left(m_{0}\right)}$ are fixed in such a way that $I_{3}^{(1)}, \ldots, I_{3}^{\left(m_{0}\right)}$ are distinct, and $I_{3}^{(i)} \neq X^{(j)}$ for $1 \leq \forall i \leq m_{0}$ and $1 \leq \forall j \leq m_{1}$.

Number of $h_{2}$. Similarly, the number of $h_{2}$ which satisfies

$$
\left.\begin{array}{l}
1 \leq \forall i<\forall j \leq m_{0}, \quad h_{2}\left(x_{R}^{(i)}\right) \oplus I_{3}^{(i)} \neq h_{2}\left(x_{R}^{(j)}\right) \oplus I_{3}^{(j)}, \\
1 \leq \forall i \leq m_{0}, 1 \leq \forall j \leq m_{1}, \quad h_{2}\left(x_{R}^{(i)}\right) \oplus I_{3}^{(i)} \neq X^{(j)}, \\
1 \leq \forall i, \forall j \leq m_{0}, \quad h_{2}\left(x_{R}^{(i)}\right) \oplus I_{3}^{(i)} \neq I_{3}^{(j)}, \text { and } \\
1 \leq \forall i, \forall j \leq m_{0}, \quad h_{2}\left(x_{R}^{(i)}\right) \oplus I_{3}^{(i)} \neq y_{R}^{(j)}
\end{array}\right\}
$$

is at least $\# H_{n}-\epsilon\left(\begin{array}{c}m_{0} \\ 2\end{array}\right) \# H_{n}-\frac{m_{0} m_{1} \# H_{n}}{2^{n}}-\frac{2 m_{0}^{2} \# H_{n}}{2^{n}}$. Fix $h_{2}$ which satisfies (16) arbitrarily. This implies that $I_{4}^{(1)}, \ldots, I_{4}^{\left(m_{0}\right)}$ are fixed in such a way that:

$-I_{4}^{(1)}, \ldots, I_{4}^{\left(m_{0}\right)}$ are distinct,

$-I_{4}^{(i)} \neq X^{(j)}$ for $1 \leq \forall i \leq m_{0}$ and $1 \leq \forall j \leq m_{1}$, and

$-I_{4}^{(i)} \neq I_{3}^{(j)}$ and $I_{4}^{(i)} \neq y_{R}^{(j)}$ for $1 \leq \forall i, \forall j \leq m_{0}$.

Number of $h_{3}$. Similarly, the number of $h_{3}$ which satisfies

$$
\left.\begin{array}{l}
1 \leq \forall i<\forall j \leq m_{0}, \quad h_{3}\left(y_{L}^{(i)} \oplus y_{R}^{(i)}\right) \oplus y_{R}^{(i)} \neq h_{3}\left(y_{L}^{(j)} \oplus y_{R}^{(j)}\right) \oplus y_{R}^{(j)}, \\
1 \leq \forall i<\forall j \leq m_{0}, \quad h_{3}\left(y_{L}^{(i)} \oplus y_{R}^{(i)}\right) \oplus I_{4}^{(i)} \neq h_{3}\left(y_{L}^{(j)} \oplus y_{R}^{(j)}\right) \oplus I_{4}^{(j)} \\
1 \leq \forall i \leq m_{0}, 1 \leq \forall j \leq m_{1}, \quad h_{3}\left(y_{L}^{(i)} \oplus y_{R}^{(i)}\right) \oplus I_{4}^{(i)} \neq Y^{(j)}, \\
1 \leq \forall i \leq m_{0}, 1 \leq \forall j \leq m_{1}, h_{3}\left(y_{L}^{(i)} \oplus y_{R}^{(i)}\right) \oplus y_{R}^{(i)} \neq Y^{(j)}, \text { and } \\
1 \leq \forall i, \forall j \leq m_{0}, \quad h_{3}\left(y_{L}^{(i)} \oplus y_{R}^{(i)}\right) \oplus y_{R}^{(i)} \neq h_{3}\left(y_{L}^{(j)} \oplus y_{R}^{(j)}\right) \oplus I_{4}^{(j)}
\end{array}\right\}
$$

is at least $\# H_{n}-2 \epsilon\left(\begin{array}{c}m_{0} \\ 2\end{array}\right) \# H_{n}-\frac{2 m_{0} m_{1} \# H_{n}}{2^{n}}-\epsilon m_{0}^{2} \# H_{n}$. Fix $h_{3}$ which satisfies (17) arbitrarily. This implies that $O_{4}^{(1)}, \ldots, O_{4}^{\left(m_{0}\right)}$ and $O_{3}^{(1)}, \ldots, O_{3}^{\left(m_{0}\right)}$ are fixed in such a way that:

$-O_{4}^{(1)}, \ldots, O_{4}^{\left(m_{0}\right)}$ are distinct,

$-O_{3}^{(1)}, \ldots, O_{3}^{\left(m_{0}\right)}$ are distinct,

- $O_{3}^{(i)} \neq Y^{(j)}$ for $1 \leq \forall i \leq m_{0}$ and $1 \leq \forall j \leq m_{1}$,

$-O_{4}^{(i)} \neq Y^{(j)}$ for $1 \leq \forall i \leq m_{0}$ and $1 \leq \forall j \leq m_{1}$, and

- $O_{4}^{(i)} \neq O_{3}^{(j)}$ for $1 \leq \forall i, \forall j \leq m_{0}$.

Number of $p$. Now $h_{1}, h_{2}$ and $h_{3}$ are fixed in such a way that

$$
I_{3}^{(1)}, \ldots, I_{3}^{\left(m_{0}\right)}, I_{4}^{(1)}, \ldots, I_{4}^{\left(m_{0}\right)}, X^{(1)}, \ldots, X^{\left(m_{1}\right)}
$$

(which are inputs to $p$ ) are all distinct and

$$
O_{3}^{(1)}, \ldots, O_{3}^{\left(m_{0}\right)}, O_{4}^{(1)}, \ldots, O_{4}^{\left(m_{0}\right)}, Y^{(1)}, \ldots, Y^{\left(m_{1}\right)}
$$

(which are corresponding outputs of $p$ ) are all distinct. In other words, for $p$, the above $2 m_{0}+m_{1}$ input-output pairs are determined. The other $2^{n}-2 m_{0}-m_{1}$ 
input-output pairs are undetermined. Therefore we have $\left(2^{n}-2 m_{0}-m_{1}\right)$ ! possible choices of $p$ for any such fixed $\left(h_{1}, h_{2}, h_{3}\right)$.

Then the number of $\left(h_{1}, h_{2}, p, h_{3}\right)$ which satisfy (2) is at least

$$
\begin{aligned}
& \left(\# H_{n}\right)^{3}\left(2^{n}-2 m_{0}-m_{1}\right) !\left(1-\epsilon\left(\begin{array}{c}
m_{0} \\
2
\end{array}\right)-\frac{m_{0} m_{1}}{2^{n}}\right) \\
& \quad \times\left(1-\epsilon\left(\begin{array}{c}
m_{0} \\
2
\end{array}\right)-\frac{m_{0} m_{1}}{2^{n}}-\frac{2 m_{0}^{2}}{2^{n}}\right)\left(1-2 \epsilon\left(\begin{array}{c}
m_{0} \\
2
\end{array}\right)-\frac{2 m_{0} m_{1}}{2^{n}}-\epsilon m_{0}^{2}\right) \\
& \geq\left(\# H_{n}\right)^{3}\left(2^{n}-2 m_{0}-m_{1}\right) !\left(1-\epsilon \cdot m_{0}\left(3 m_{0}-2\right)-\frac{2 m_{0}\left(m_{0}+2 m_{1}\right)}{2^{n}}\right)
\end{aligned}
$$

This concludes the proof of the lemma.

\section{Conclusion}

In this paper, we proved that:

1. $\psi\left(p_{1}, p_{2}, p_{3}, p_{4}, p_{5}\right)$ is not super-pseudorandom if the adversary is allowed to have oracle access to $\left\{p_{1}, p_{1}^{-1}\right\}$ or $\left\{p_{5}^{-1}\right\}$ (Theorem 3.1 ).

2. $\psi\left(h_{1}, h_{2}, p, p, h_{3}^{-1}\right)$ is super-pseudorandom even if the adversary has oracle access to $p$ and $p^{-1}$ (Theorem 3.2).

3. $\psi\left(h_{1}, p_{1}, p_{2}, p_{3}, h_{2}^{-1}\right)$ is super-pseudorandom even if the adversary has oracle access to $p_{1}, p_{1}^{-1}, p_{2}, p_{2}^{-1}, p_{3}$ and $p_{3}^{-1}$ (Theorem 3.3. ).

The following concrete questions remain to be tackled.

- Is it possible to distinguish $\psi\left(p_{1}, p_{2}, p_{3}, p_{4}, p_{5}\right)$ from $P_{2 n}$ with access to only one of $\left\{p_{1}, p_{1}^{-1}, p_{5}\right\}$ ?

- For example, is $\psi\left(h_{1}, p_{1}, p_{1}, p_{2}, h_{2}^{-1}\right)$ secure when the adversary has oracle access to $p_{1}, p_{1}^{-1}, p_{2}$ and $p_{2}^{-1}$ ?

\section{References}

1. J.L.Carter and M.N.Wegman. Universal classes of hash functions. JCSS, vol. 18, No. 2, pages 143-154, 1979.

2. M.Luby and C.Rackoff. How to construct pseudorandom permutations from pseudorandom functions. SIAM J. Comput., vol. 17, No. 2, pages 373-386, April 1988.

3. S.Lucks. Faster Luby-Rackoff ciphers. Fast Software Encryption, FSE '96, LNCS 1039, pages 189-203, Springer-Verlag.

4. M.Matsui. New structure of block ciphers with provable security against differential and linear cryptanalysis. Fast Software Encryption, FSE '96, LNCS 1039, pages 206-218, Springer-Verlag.

5. M.Matsui. New block encryption algorithm MISTY. Fast Software Encryption, FSE '97, LNCS 1267, pages 54-68, Springer-Verlag.

6. M.Naor and O.Reingold. On the construction of pseudorandom permutations: Luby-Rackoff revised. J. Cryptology, vol. 12, No. 1, pages 29-66, Springer-Verlag, 1999. 
7. K.Nyberg and L.R.Knudsen. Provable security against a differential attacks. J. Cryptology, vol. 8, No. 1, pages 27-37, Springer-Verlag, 1995.

8. J.Patarin. Pseudorandom permutations based on the DES scheme. Proceedings of Eurocode '90, LNCS 514, pages 193-204, Springer-Verlag, 1990.

9. J.Patarin. New results of pseudorandom permutation generators based on the DES scheme. Advances in Cryptology - CRYPTO '91, LNCS 576, pages 301-312, Springer-Verlag, 1991.

10. Z.Ramzan and L.Reyzin. On the round security of symmetric-key cryptographic primitives. Advances in Cryptology - CRYPTO 2000, LNCS 1880, pages 376-393, Springer-Verlag, 2000.

11. K.Sakurai and Y.Zheng. On non-pseudorandomness from block ciphers with provable immunity against linear cryptanalysis. IEICE Trans. fundamentals, vol. E80A, No. 1, pages 19-24, April 1997.

12. M.Sugita. Pseudorandomness of a block cipher MISTY. Technical report of IEICE, ISEC 96-9, pages 13-21, 1996.

13. M.Sugita. Pseudorandomness of block ciphers MISTY1. Technical report of IEICE, ISEC 97-19, pages 53-64, 1997.

14. M.N.Wegman and J.L.Carter. New hash functions and their use in authentication and set equality. JCSS, vol. 22, No. 3, pages 265-279, 1981. 